\title{
Post-lumpectomy CT-guided tumor bed delineation for breast boost and partial breast irradiation: Can additional pre- and postoperative imaging reduce interobserver variability?
}

\author{
MARISKA D. DEN HARTOGH, MARIELLE E.P. PHILIPPENS, IRIS E. VAN DAM, CATHARINA E. KLEYNEN, \\ ROBBERT J.H.A. TERSTEEG, ALEXIS N.T.J. KOTTE, MARCO VAN VULPEN, \\ BRAM VAN ASSELEN and DESIRÉE H.J.G. VAN DEN BONGARD
}

Department of Radiation Oncology, University Medical Center Utrecht, Utrecht, The Netherlands

Received August 7, 2014; Accepted April 20, 2015

DOI: 10.3892/ol.2015.3697

\begin{abstract}
For breast boost radiotherapy or accelerated partial breast irradiation, the tumor bed (TB) is delineated by the radiation oncologist on a planning computed tomography (CT) scan. The aim of the present study was to investigate whether the interobserver variability (IOV) of the TB delineation is reduced by providing the radiation oncologist with additional magnetic resonance imaging (MRI) or CT scans. A total of 14 T1-T2 breast cancer patients underwent a standard planning $\mathrm{CT}$ in the supine treatment position following lumpectomy, as well as additional pre- and postoperative imaging in the same position. Post-lumpectomy TBs were independently delineated by four breast radiation oncologists on standard postoperative $\mathrm{CT}$ and on $\mathrm{CT}$ registered to an additional imaging modality. The additional imaging modalities used were postoperative MRI, preoperative contrast-enhanced (CE)-CT and preoperative CE-MRI. A cavity visualization score (CVS) was assigned to each standard postoperative CT by each observer. In addition, the conformity index $(\mathrm{CI})$, volume and distance between centers of mass (dCOM) of the TB delineations were calculated. On CT, the median CI was 0.57 , with a median volume of $22 \mathrm{~cm}^{3}$ and dCOM of $5.1 \mathrm{~mm}$. The addition of postoperative MRI increased the median TB volume significantly to $28 \mathrm{~cm}^{3}$ $(\mathrm{P}<0.001)$, while the $\mathrm{CI}(\mathrm{P}=0.176)$ and $\mathrm{dCOM}(\mathrm{P}=0.110)$ were not affected. The addition of preoperative $\mathrm{CT}$ or MRI increased the TB volume to 26 and $25 \mathrm{~cm}^{3}$, respectively (both $\mathrm{P}<0.001$ ), while the $\mathrm{CI}$ increased to 0.58 and 0.59 (both $\mathrm{P}<0.001)$ and the $\mathrm{dCOM}$ decreased to $4.7 \mathrm{~mm}(\mathrm{P}=0.004)$ and $4.6 \mathrm{~mm}(\mathrm{P}=0.001)$, respectively. In patients with $\mathrm{CVS} \leq 3$, the median CI was 0.40 on CT, which was significantly increased
\end{abstract}

Correspondence to: Dr Mariska D. den Hartogh, Department of Radiation Oncology, University Medical Center Utrecht, P.O. Box 85500, Utrecht 3508 GA, The Netherlands

E-mail: m.denhartogh-3@umcutrecht.nl

Key words: breast-conserving therapy, radiotherapy, tumor bed, interobserver variability, magnetic resonance imaging by all additional imaging modalities, up to 0.52 , and was accompanied by a median volume increase up to $6 \mathrm{~cm}^{3}$. In conclusion, the addition of postoperative MRI, preoperative CE-CT or preoperative CE-MRI did not result in a considerable reduction in the IOV in postoperative CT-guided TB delineation, while target volumes marginally increased. The value of additional imaging may be dependent on CVS.

\section{Introduction}

Radiotherapy following breast-conserving surgery is an integral part of breast-conserving treatment in early-stage breast cancer (1). Traditionally, the entire breast is irradiated, with an additional boost dose to the tumor bed (TB) in patients with a higher risk of local recurrence (2). Although the geometric precision of modern radiation dose delivery is high, target delineation uncertainties are often large (3). In particular, delineation of the TB area, which is performed on the postoperative radiotherapy planning computed tomography (CT) scan, is highly variable among radiation oncologists (4-8). This may potentially lead to treatment inaccuracies, which are of particular concern due to the increasing use of Accelerated Partial Breast Irradiation (APBI), in which only the breast tissue surrounding the TB is irradiated. Furthermore, postoperative seroma formation may lead to large TB volumes, which are associated with an increased risk of subcutaneous fibrosis and poorer cosmetic results (9-12).

There are a number of potential options to improve TB visualization for standard CT-guided TB delineation. Magnetic resonance imaging (MRI) may have additional value due to its excellent soft-tissue contrast compared with $\mathrm{CT}$. The use of different MRI sequences makes it possible to differentiate between fibroglandular tissue, fluid and fat, while it can also detect the heterogeneity and irregularity of seromas (13). Another potential strategy for improving CT-guided TB delineation is to increase the observer's knowledge of the original tumor location. Currently, the preoperative diagnostic mammogram or MRI scan are used by the radiation oncologist to reconstruct the original tumor location on the planning CT scan. This diagnostic imaging is not acquired in the supine radiotherapy treatment position, which presents challenges in 
Table I. Magnetic resonance imaging sequence parameters.

\begin{tabular}{lccc}
\hline Parameter & Postoperative & Postoperative & Preoperative dynamic \\
T2 & T1 Dixon FFE & Transverse \\
\hline Orientation & Transverse & Transverse & $3 \mathrm{D}$ \\
Acquisition mode & $3 \mathrm{D}$ & $3 \mathrm{D}$ & $250 \times 448 \times 200$ \\
FOV, mm & $250 \times 450 \times 200$ & $250 \times 448 \times 200$ & $208 \times 388 \times 167$ \\
Matrix size & $200 \times 357 \times 167$ & $252 \times 447 \times 182$ & $1.20 \times 1.21 \times 2.40$ \\
Acquired voxel size, $\mathrm{mm}$ & $1.25 \times 1.25 \times 2.40$ & $0.99 \times 1.00 \times 2.20$ & $1.16 \times 1.18 \times 1.20$ \\
Reconstructed voxel size, $\mathrm{mm}$ & $0.78 \times 0.78 \times 1.2$ & $0.95 \times 0.93 \times 1.10$ & $6.1 / 1.87$ \\
TR/TE, ms/ms & $2000 / 172$ & $7.1 / 1.71$ & 10 \\
Flip angle & 90 & 12 & NA \\
Refocusing angle & 120 & $\mathrm{NA}$ & 84 \\
Turbo factor & 74 & 117 & 1 \\
NSA & 1 & 2 & Dixon \\
Fat suppression & SPAIR & Dixon & $4: 13$ \\
Acquisition time, min & $5: 40$ & $7: 51$ &
\end{tabular}

TSE, turbo spin echo; SPAIR, spectral adiabatic inversion recovery; FFE, fast field echo; 3D, three-dimensional; FOV, field of view; TR, repetition time; TE, echo time; NSA, number of averages; NA, not applicable.

interpreting the original tumor position on the supine planning CT scan. Preoperative imaging acquired in the treatment position may improve the observer's knowledge regarding the original tumor location and, thereby, potentially reduce interobserver variability (IOV). The addition of a preoperative contrast-enhanced (CE)-CT in the supine radiotherapy treatment position to the standard postoperative planning CT was investigated by Boersma et al (14), who reported a minor reduction of the IOV. However, in our recent delineation study on preoperative breast tumor delineation, CE-MRI in the radiotherapy supine position was demonstrated to be superior to CE-CT for tumor detection and the visualization of tumor irregularities and spiculations (15). Therefore, in the current study, CE-CT, which is most commonly available in radiotherapy institutes, and CE-MRI were investigated as additional imaging modalities with which to improve post-lumpectomy CT-guided TB definition.

The aim of the present study was to investigate whether the IOV of standard CT-guided post-lumpectomy TB delineation is reduced through the use of additional postoperative MRI, preoperative CE-CT or preoperative CE-MRI in the supine radiotherapy treatment position.

\section{Materials and methods}

Patients and selection. The study population included NTR3198 study patients (15), who had received CT and MRI scans prior to and following lumpectomy as part of the study to quantify pre- and postoperative treatment volumes. This study was approved by the institutional review committee of the University Medical Center Utrecht (Utrecht, The Netherlands) and registered in the International Clinical Trials Registry. Written informed consent was obtained from all patients. Patients eligible for inclusion had a clinical TNM T1-T2, N0-staged adenocarcinoma of the breast (16), and were scheduled for breast-conserving therapy. Patients with lobular carcinoma, a history of ipsilateral breast surgery, contraindications for 1.5 Tesla MRI or iodine allergy, and patients who received neoadjuvant treatment, were not eligible. Patients were enrolled between November 2011 and November 2012.

Image acquisition. The eligible patients underwent preoperative CE-CT and CE-MRI scans prior to lumpectomy, and a standard planning CT scan directly followed by an MRI scan at a median of 21 days (range, 14-50 days) after lumpectomy. All scans were performed in the supine treatment position.

For CT and MRI, the patients were placed with the arms in abduction and hands above the head at $10^{\circ}$ inclination and with the use of a knee support (C-Qual ${ }^{\mathrm{TM}}$ and Thorawedge for CT and MRI, respectively; CIVCO Medical Solutions, Reeuwijk, The Netherlands). The tumor or surgical scar was marked on the skin with a CT/MRI compatible wire.

For MRI, a wide bore $(70 \mathrm{~cm})$ MRI scanner (Ingenia 1.5T; Philips Medical Systems, Best, The Netherlands) and anterior receive coil were used. To prevent breast deformation by the anterior receive coil, a polymethyl methacrylate support was designed, which was adjustable to patient habitus and breast size. The following three-dimensional high resolution MRI images were used in this study: T1 weighted (T1w) fast field echo (FFE) with and without fat suppression (Dixon), T2 weighted (T2w) turbo spin echo (TSE) with fat suppression [spectral adiabatic inversion recovery (SPAIR)] and preoperative dynamic series of CE T1w Dixon images (17). The MRI sequence parameters are provided in Table I. The total acquisition times of the pre- and postoperative MRI protocols were 21 and $14 \mathrm{~min}$, respectively.

Target volume delineation. All the images were transferred to the in-house developed delineation software (18). The postoperative MRI was registered to the postoperative planning CT 
Table II. Patient characteristics $(n=14)$.

\begin{tabular}{|c|c|}
\hline Characteristic & Value \\
\hline \multicolumn{2}{|l|}{ Age, years } \\
\hline Median & 61 \\
\hline Range & $48-70$ \\
\hline \multicolumn{2}{|l|}{ Microscopic tumor diameter, $\mathrm{mm}$} \\
\hline Median & 12 \\
\hline Range & $6-29$ \\
\hline \multicolumn{2}{|l|}{ Histology, $\mathrm{n}$} \\
\hline Ductal carcinoma & 10 \\
\hline Ductal-lobular carcinoma & 3 \\
\hline Tubular carcinoma & 1 \\
\hline \multicolumn{2}{|l|}{ Side, $\mathrm{n}$} \\
\hline Left & 7 \\
\hline Right & 7 \\
\hline \multicolumn{2}{|l|}{ Time between surgery and postoperative imaging, days } \\
\hline Median & 21 \\
\hline Range & $14-50$ \\
\hline \multicolumn{2}{|l|}{ Surgical technique, $\mathrm{n}$} \\
\hline Open cavity & 2 \\
\hline Full thickness closure & 12 \\
\hline \multicolumn{2}{|l|}{ Number of clips placed } \\
\hline Median & 5 \\
\hline Range & $4-6$ \\
\hline \multicolumn{2}{|l|}{ Cavity visualization scores, $\mathrm{n}$} \\
\hline 1 - no cavity visible & 1 \\
\hline 2 - heterogeneous cavity, indistinct margins & 5 \\
\hline 3 - heterogeneous cavity, some distinct margins & 2 \\
\hline 4 - mildly heterogenous cavity, mostly distinct margins & s 5 \\
\hline 5 - homogenous cavity, clearly identified margins & 1 \\
\hline Mean score & 3 \\
\hline
\end{tabular}

by rigid mutual information registration on a box around the tumor, using the T1w images with fat-suppression. The preoperative CE-CT and CE-MRI were registered to the planning CT by automatic registration on the chest wall. Subsequently, four experienced breast radiation oncologists independently delineated the TB, using written delineation instructions. These instructions were formulated in a consensus meeting with all observers, supervised by an experienced breast radiologist. The consensus meeting was repeated once, to answer questions regarding MRI and to discuss ambiguities in the delineation instructions. Delineation took place as follows: Firstly, the observer delineated the TB on standard postoperative planning $\mathrm{CT}$ and assigned a cavity visualization score (CVS) of 1 (no cavity visible), 2 (heterogeneous cavity with indistinct margins), 3 (heterogeneous cavity with some distinct margins), 4 (mildly heterogeneous cavity with mostly distinct margins), or 5 (homogeneous cavity with clearly identified margins) (19). Next, the CT-based delineation was duplicated and adjusted according to findings of the co-registered postoperative MRI, preoperative CT and preoperative MRI. The original CT-guided delineation was duplicated to prevent influencing the data by intraobserver variations and to solely study the influence of additional imaging on this standard delineation method.

Data analysis. The conformity index (CI) and distance between the centers of mass (dCOM) of the TB contours were calculated for all the possible observer pairs. The CI per observer pair was calculated using the following formula: $\mathrm{CI}=$ volume of agreement / total encompassing volume. Consequently, a CI of 1 implies a perfect agreement among observers, while a CI of 0 indicates no overlap in delineations. For dCOM, a value of 0 indicates that two delineations are centered at the same position.

Median values and accompanying ranges were used to describe the data, as not all variables were normally distributed. A Wilcoxon signed-rank test was performed to compare paired variables using SPSS version 20 (IBM SPSS, Armonk, NY, USA) with a significance level of 0.05 . To visualize the effect of additional imaging on the CI, the change in $\mathrm{CI}$ per observer pair was plotted against the original CI of that observer pair on CT using GraphPad Prism 6 (GraphPad Software, Inc., La Jolla, CA, USA). Furthermore, these outcomes were categorized as $\mathrm{CVS} \leq 3$ and $\mathrm{CVS} \geq 4$.

\section{Results}

Patients. A total of 14 patients were prospectively included in the present study (Table II). The majority of patients underwent full thickness closure of the excision cavity, which consisted of suturing the deep and superficial layers of the cavity's breast tissue. A representative example of standard postoperative $\mathrm{CT}$, registered to postoperative MRI, preoperative CE-CT and preoperative CE-MRI in one of the patients is shown in Fig. 1. The different features of the TB as visualized by different MRI sequences are shown in Fig. 2. Fat suppressed T1w (Fig. 2B) and T2w (Fig. 2D) images enable distinction between fibroglandular tissue and seroma. Surgical clips can be visualized by the T1w images without fat-suppression (Fig. 2C).

IOV in volume and $\mathrm{dCOM}$. TB delineation determined by the four independent observers resulted in wide ranges in volume, CI and dCOM on standard postoperative planning CT (Table III), which did not improve with any of the additional imaging methods. The lowest limit of the CI range (CI, 0.00) was observed in cases with an absolute disagreement among observers, with no overlap of TB delineations. This disagreement occurred in one patient with a centrally located TB and a CVS of 1 (no cavity visible), assigned unanimously by all observers. One observer contoured a region different from the other three observers. Excluding this outlier from the analysis did not influence the outcomes. This observer did not cause outliers in any of the other patients. Data analysis revealed that none of the observers deviated from the other observers with regard to volume, $\mathrm{CI}$ and $\mathrm{dCOM}$.

Addition of a postoperative MRI to the standard postoperative planning CT did not influence the $\mathrm{CI}(\mathrm{P}=0.176)$ or $\mathrm{dCOM}$ $(\mathrm{P}=0.110$; Table III). However, the TB volumes increased significantly $(\mathrm{P}<0.001)$ with a median increase of $6 \mathrm{~cm}^{3}$.

Addition of a preoperative CT or MRI significantly increased the $\mathrm{CI}(\mathrm{P}<0.001$ for both $)$ and $\mathrm{dCOM}(\mathrm{P}=0.004$ and 
Table III. Volume, conformity index and dCOM of the tumor bed delineations.

\begin{tabular}{|c|c|c|c|}
\hline Parameter & Median & Range & $P$-value \\
\hline \multicolumn{4}{|l|}{ Volume, $\mathrm{cm}^{3}$} \\
\hline $\mathrm{CT}$ & 22 & 4-934 & \\
\hline $\mathrm{CT}+$ postoperative MRI & 28 & $3-964$ & $<0.001$ \\
\hline $\mathrm{CT}+$ preoperative $\mathrm{CT}$ & 26 & $6-933$ & $<0.001$ \\
\hline $\mathrm{CT}+$ preoperative $\mathrm{MRI}$ & 25 & $7-933$ & $<0.001$ \\
\hline \multicolumn{4}{|l|}{ Conformity index } \\
\hline $\mathrm{CT}$ & 0.57 & $0.00-0.90$ & \\
\hline $\mathrm{CT}+$ postoperative MRI & 0.61 & $0.00-0.89$ & 0.176 \\
\hline $\mathrm{CT}+$ preoperative $\mathrm{CT}$ & 0.58 & $0.00-0.90$ & $<0.001$ \\
\hline $\mathrm{CT}+$ preoperative MRI & 0.59 & $0.00-0.89$ & $<0.001$ \\
\hline \multicolumn{4}{|l|}{$\mathrm{dCOM}, \mathrm{mm}$} \\
\hline $\mathrm{CT}$ & 5.11 & $0-53$ & \\
\hline $\mathrm{CT}+$ postoperative MRI & 3.72 & $0-52$ & 0.110 \\
\hline $\mathrm{CT}+$ preoperative $\mathrm{CT}$ & 4.69 & $1-42$ & 0.004 \\
\hline $\mathrm{CT}+$ preoperative MRI & 4.56 & $0-48$ & 0.001 \\
\hline
\end{tabular}

aP-value refers to differences in the median values obtained using an additional imaging modality compared with the standard postoperative CT. CT, computed tomography; MRI, magnetic resonance imaging; dCOM, distance between centers of mass.
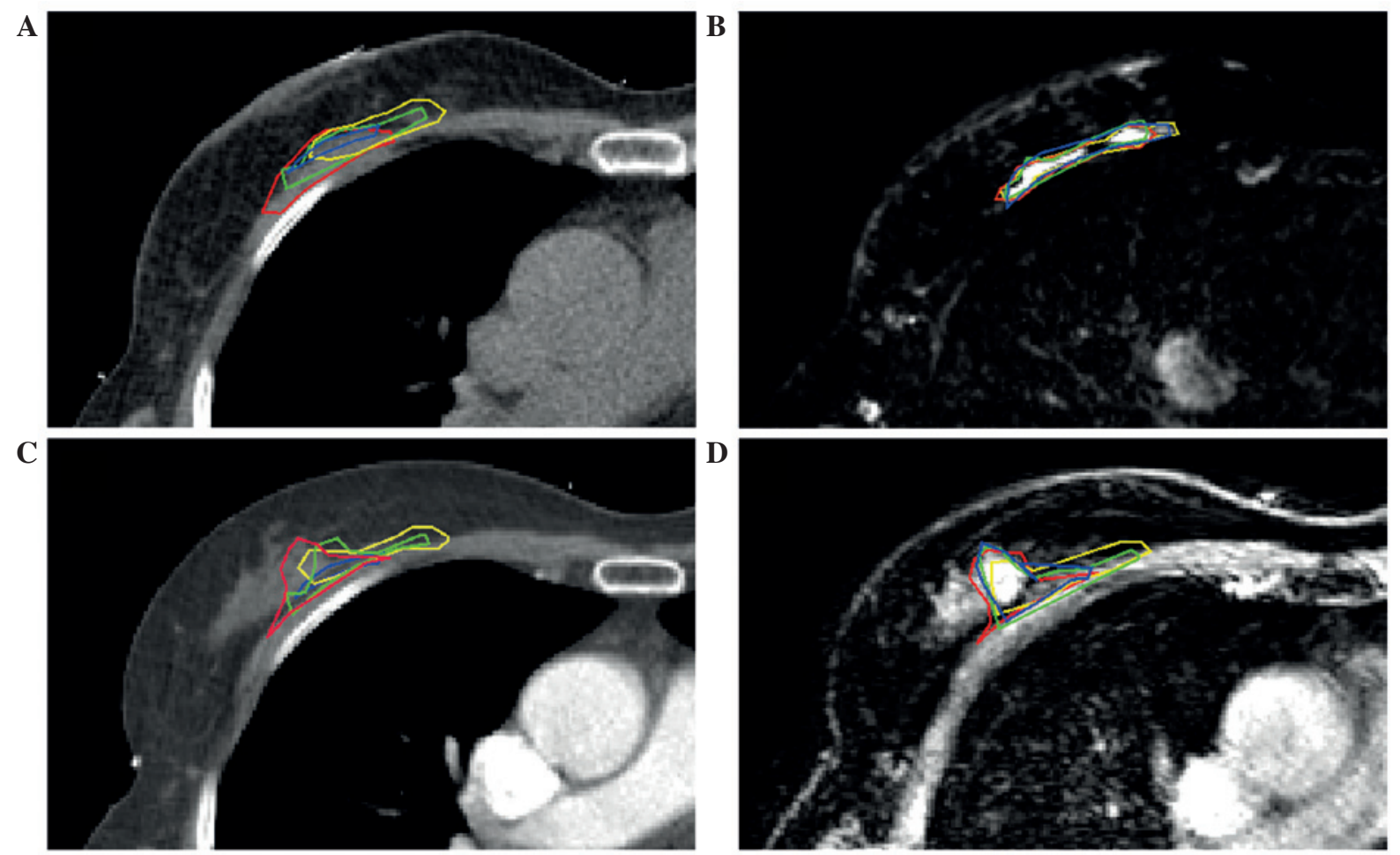

Figure 1. CT and MRI scans of a 64-year-old patient with pT1cN0(sn) ductal carcinoma of the right breast. The cavity visualization score was 2 on CT. CT-guided tumor bed delineation was determined by four observers, demonstrated in different colors, on the same transverse slice on (A) postoperative CT, (B) postoperative T2-weighted MRI, (C) preoperative CE-CT and (D) preoperative CE-MRI scans. CT, computed tomography; MRI, magnetic resonance imaging; CE, contrast enhanced.

$\mathrm{P}=0.001$, respectively; Table III). A statistically significant absolute volume increase of $4 \mathrm{~cm}^{3}$ and $3 \mathrm{~cm}^{3}$, was observed following the addition of preoperative CE-CT and CE-MRI, respectively (both $\mathrm{P}<0.001$ ).
The change in CI per observer pair following the addition of an imaging method was plotted against the original CI of that observer pair on standard postoperative CT (Fig. 3). These outcomes were categorized as $\mathrm{CVS} \leq 3$ and $\mathrm{CVS} \geq 4$. 

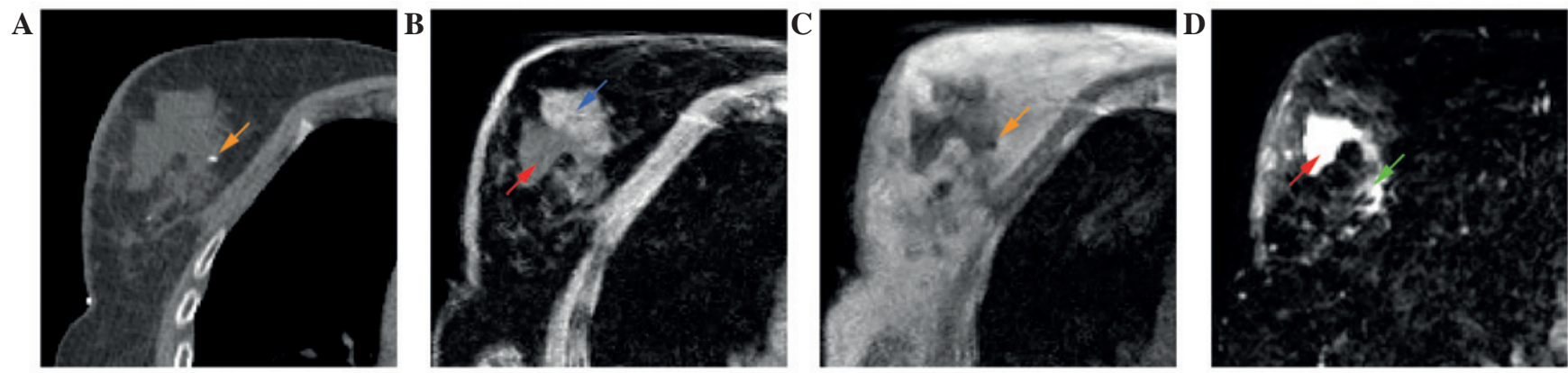

Figure 2. A 62-year-old patient with pT1cNO(sn) ductal carcinoma of the right breast. Different features of the tumor bed as shown on (A) postoperative planning CT, (B) postoperative T1w MRI with fat suppression, (C) postoperative T1w MRI and (D) postoperative T2w MRI with fat suppression. Arrows: Blue, fibroglandular tissue; red, seroma; orange, surgical clip; green, area with intermediate signal intensity. CT, computed tomography; MRI, magnetic resonance imaging; T1w, T1 weighted; T2w, T2 weighted.
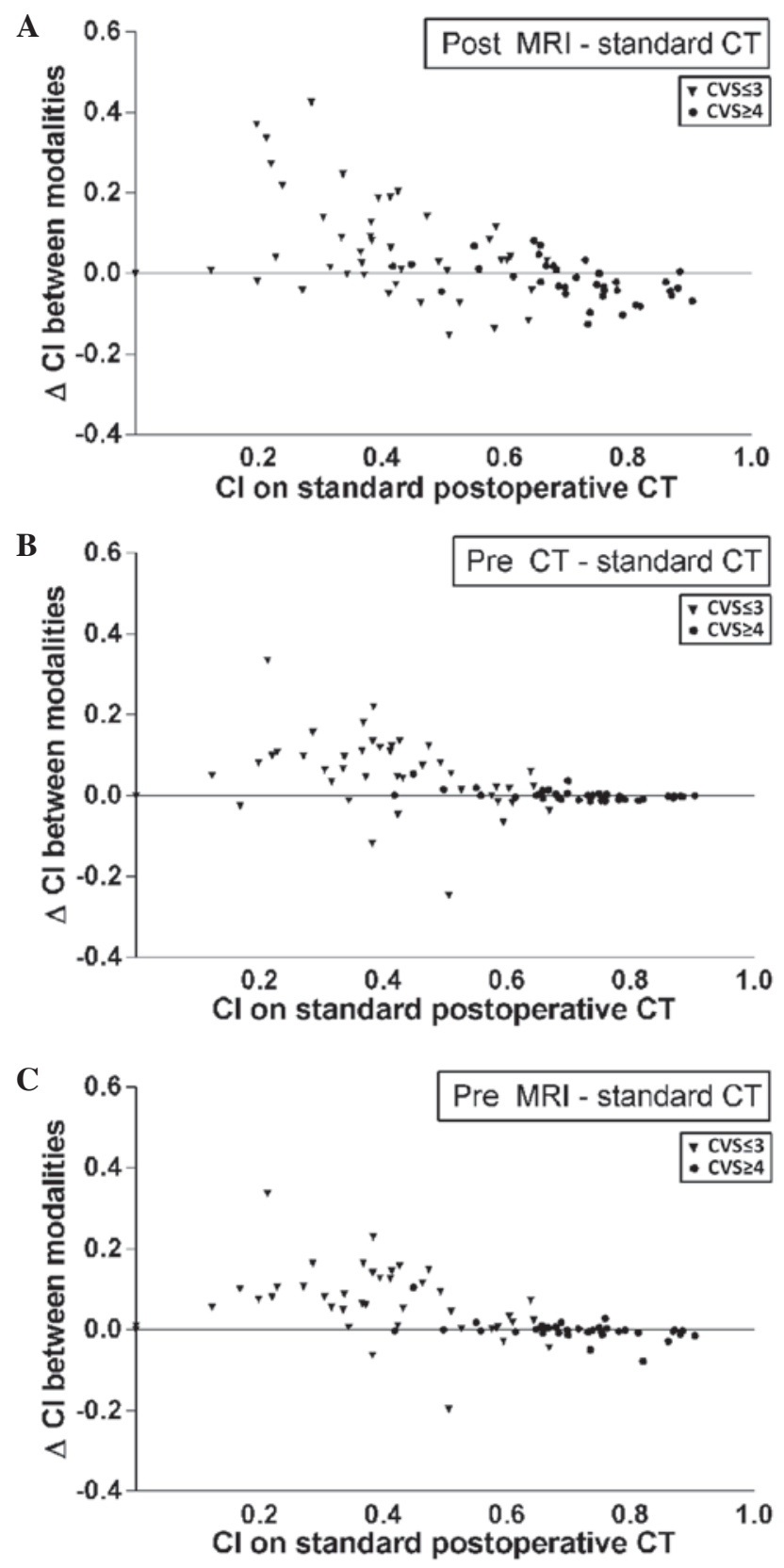

Figure 3. CI per observer pair on standard postoperative CT (x-axis), plotted against the change $(\Delta)$ in $\mathrm{CI}$ ( $\mathrm{y}$-axis) after addition of (A) postoperative MRI, (B) preoperative CT, and (C) preoperative MRI. CI, conformity index; $\mathrm{CT}$, computed tomography; MRI, magnetic resonance imaging; CVS, cavity visualization score.
In general, the CI was higher in patients with a high CVS score (Fig. 3, circles). In these patients, conformity did not increase with the use of additional imaging modalities and significantly decreased following the addition of postoperative MRI $(\mathrm{P}=0.016)$. By contrast, in patients with a low $\mathrm{CI}$ and a more heterogeneous TB (CVS $\leq 3)$, an increase in CI was observed, from a median of 0.40 on CT to 0.52 on CT with additional preoperative MRI. In this same subgroup, volumes also increased, from $17 \mathrm{~cm}^{3}$ on CT to $23 \mathrm{~cm}^{3}$ on CT with additional postoperative MRI.

\section{Discussion}

To the best of our knowledge, the present study was the first to investigate the value of additional pre- and postoperative CT and MRI, with all imaging acquired in the radiotherapy supine position using wide bore CT and MRI scanners, and its effect on the IOV of the TB delineation.

The results indicated that the addition of postoperative MRI did not improve the IOV of standard CT-guided TB delineation. This was unexpected, as the use of different MRI sequences enables differentiation between various soft tissues (Fig. 2). In the fat-suppressed images, MRI is unique in its clear contrast between seroma and fibroglandular tissue. However, the interpretation of the different MRI sequences in combination with the available patient information appears to be observer-dependent, despite the training and written delineation instructions. The observers were found to expand their target volume rather than reduce their original CT-guided delineation based on the information provided by additional imaging. For instance, in Fig. 1B, the area of seroma and architectural distortion on T2w MRI was included. Furthermore, when the observers were provided with preoperative imaging, they expanded the original CT-based delineation in the direction of the original tumor, and did not adjust, for instance, the medial borders. It appears that observers will expand their contour based on additional information, but are unlikely to reduce it when an area may not be part of the TB. In that case, they seem to favor their interpretation of the standard planning $\mathrm{CT}$, which they are most familiar with. This finding may also explain the volume increase that is observed subsequent to providing additional imaging.

The heterogeneity in CI, as shown in Fig. 3, indicates that the subgroup of patients with CVS $\leq 3$ potentially benefits from 
the use of MRI, since the CI mostly increases in this subgroup. However, the clinical relevance of this finding is debatable as the median CI following the addition of preoperative MRI for this patient subgroup was only 0.52 . Furthermore, these findings must be interpreted with caution, as delineated volumes also increased by a median value of up to $6 \mathrm{~cm}^{3}$. It may be of interest to focus on the subgroup of patients with a CVS of $\leq 3$ in future studies in a larger patient cohort, particularly as a higher incidence of these lower CVS scores may be expected with the increasing use of full-thickness closure following lumpectomy.

In line with the results of the present study, Kirby et al (20) reported increased TB volumes delineated on CT-MRI datasets. The current study investigated whether additional MRI, registered to the standard postoperative planning $\mathrm{CT}$, was able to reduce the IOV of TB delineation. In a previous study investigating TB delineation using MRI-only, the conformity among observers was even lower (21), which is also consistent with the results reported by Giezen et al (22). However, in a study by Jolicoeur et al (23), the IOV improved and the volumes were smaller on MRI-only, compared with that of CT-based delineation. This contradiction may be due to the MRI quality and the definition of the TB. In the study by Jolicoeur et al (23), the TB was defined as an architectural change on primarily T2W MRI. By contrast, in other studies, the TB was reconstructed according to architectural changes, original tumor location on preoperative diagnostic imaging, physical examination and the placement of surgical clips $(20,22)$. Furthermore, Jolicoeur et al (23) primarily used T2w sequences, while the present study used multiple sequences. The use of different surgical techniques may also have influenced the differences in outcomes between these studies: The majority of patients in the present study underwent full thickness closure, while Jolicoeur et al (23) excluded patients who underwent oncoplastic techniques, which is likely to have included full thickness closure. Subsequent to suturing the cavity walls, seroma may follow the suturing lines, the shapes of which may be more subject to interpretational differences compared with clearly defined cavity walls in superficially closed cavities (Fig. 2, red and green arrows).

Giezen et al (22) and Jolicoeur et al (23) investigated TB delineation using MRI separately and compared it with CT-guided delineation. In the present study, the additional value of MRI registered to standard postoperative CT was assessed, as this would be the application in clinical practice. In this setting, no added value of postoperative MRI was observed for the general postoperative patient population.

Despite statistically significant differences, no clinically relevant effects of either additional preoperative MRI or CT imaging on the CI of postoperative CT-guided TB delineation were observed in the current study. These findings are consistent with those of Boersma et al (14), who found no increase in CI following the addition of a preoperative CE-CT.

The study results may be influenced by structural observer outliers, observer training and observer knowledge of MRI interpretation. As there is no gold standard with which to validate the 'correct' imaging modality for TB delineation, consensus among observers is used as an alternative method. Upon the inspection of the data, no observer deviated with regard to volume, $\mathrm{CI}$ or dCOM. Even in the presence of guidelines, training and an adequate number of clips, considerable variation exists (24). A possible limitation of the current study is the small number of patients used. However, with increases and decreases in IOV and volumes, a larger cohort is unlikely to alter the average difference considerably.

For the overall patient population, additional imaging will not improve consistency in TB delineation. However, it may be possible to further improve the IOV of postoperative CT-guided TB delineation. A number of studies have proposed irradiation of the high-risk breast tissue surrounding the tumor prior to lumpectomy, while the tumor remains in situ $(15,25-27)$. This preoperative approach would result in a markedly lower IOV compared with the current standard postoperative treatment $(15,27)$. Furthermore, preoperative image-guided target volume definition may be validated using pathological studies as a gold standard, which may improve confidence in an accurate treatment of the high-risk area (28).

In conclusion, the addition of postoperative MRI, preoperative $\mathrm{CE}-\mathrm{CT}$ or preoperative $\mathrm{CE}-\mathrm{MRI}$ did not result in a considerable reduction of the IOV of postoperative CT-guided TB delineation, while target volumes increased marginally. The influence of additional imaging may be dependent on CVS.

\section{Acknowledgements}

This article is part of the $\mathrm{PhD}$ thesis entitled 'Towards MRI-guided radiotherapy in early-stage breast cancer patients' (29).

\section{References}

1. Darby S, McGale P, Correa C, et al; Early Breast Cancer Trialists' Collaborative Group (EBCTCG): Effect of radiotherapy after breast-conserving surgery on 10-year recurrence and 15-year breast cancer death: Meta-analysis of individual patient data for 10,801 women in 17 randomised trials. Lancet 378: 1707-1716, 2011.

2. Bartelink H, Horiot JC, Poortmans PM, et al: Impact of a higher radiation dose on local control and survival in breast-conserving therapy of early breast cancer: 10 -year results of the randomized boost versus no boost EORTC 22881-10882 trial. J Clin Oncol 25: 3259-3265, 2007.

3. Malinen E and Muren LP: Image guided therapy - do we get the picture? Acta Oncol 53: 3-5, 2014.

4. Struikmans H, Wárlám-Rodenhuis C, Stam T, Stapper G, Tersteeg RJ, Bol GH and Raaijmakers CP: Interobserver variability of clinical target volume delineation of glandular breast tissue and of boost volume in tangential breast irradiation. Radiother Oncol 76: 293-299, 2005.

5. Coles CE, Wilson CB, Cumming J, Benson JR, Forouhi P, Wilkinson JS, Jena R and Wishart GC: Titanium clip placement to allow accurate tumour bed localisation following breast conserving surgery: Audit on behalf of the IMPORT Trial Management Group. Eur J Surg Oncol 35: 578-582, 2009.

6. Hurkmans C, Admiraal M, van der Sangen M and Dijkmans I: Significance of breast boost volume changes during radiotherapy in relation to current clinical interobserver variations. Radiother Oncol 90: 60-65, 2009.

7. van Mourik AM, Elkhuizen PH, Minkema D, Duppen JC and van Vliet-Vroegindeweij C; Dutch Young Boost Study Group: Multiinstitutional study on target volume delineation variation in breast radiotherapy in the presence of guidelines. Radiother Oncol 94: 286-291, 2010.

8. Landis DM, Luo W, Song J, Bellon JR, Punglia RS, Wong JS, Killoran JH, Gelman R and Harris JR: Variability among breast radiation oncologists in delineation of the postsurgical lumpectomy cavity. Int J Radiat Oncol Biol Phys 67: 1299-1308, 2007.

9. den Hartogh MD, van Asselen B, Monninkhof EM, et al: Excised and irradiated volumes in relation to the tumor size in breast-conserving therapy. Breast Cancer Res Treat 129: $857-865,2011$. 
10. Collette S, Collette L, Budiharto T, et al; EORTC Radiation Oncology Group: Predictors of the risk of fibrosis at 10 years after breast conserving therapy for early breast cancer: A study based on the EORTC Trial 22881-10882 'boost versus no boost'. Eur J Cancer 44: 2587-2599, 2008.

11. Vrieling C, Collette L, Fourquet A, et al; EORTC Radiotherapy and Breast Cancer Cooperative Groups: The influence of patient, tumor and treatment factors on the cosmetic results after breast-conserving therapy in the EORTC 'boost vs. no boost' trial. Radiother Oncol 55: 219-232, 2000.

12. Mukesh MB, Barnett G, Cumming J, Wilkinson JS, Moody AM, Wilson C, Wishart GC and Coles CE: Association of breast tumour bed seroma with post-operative complications and late normal tissue toxicity: Results from the Cambridge Breast IMRT trial. Eur J Surg Oncol 38: 918-924, 2012.

13. Whipp EC and Halliwell M: Magnetic resonance imaging appearances in the postoperative breast: The clinical target volume-tumor and its relationship to the chest wall. Int J Radiat Oncol Biol Phys 72: 49-57, 2008

14. Boersma LJ, Janssen T, Elkhuizen PH, Poortmans P, van der Sangen M, Scholten AN, Hanbeukers B, Duppen JC, Hurkmans $C$ and van Vliet C: Reducing interobserver variation of boost-CTV delineation in breast conserving radiation therapy using a pre-operative CT and delineation guidelines. Radiother Oncol 103: 178-182, 2012.

15. den Hartogh MD, Philippens ME, van Dam IE, et al: MRI and CT imaging for preoperative target volume delineation in breast-conserving therapy. Radiat Oncol 9: 63, 2014

16. Sobin LH, Gospodarowicz MK, Wittekind C (eds); International Union Against Cancer (UICC): TNM Classification of Malignant Tumors. 7th edition. Wiley-Blackwell, Chichester, UK, 2009.

17. Dixon WT: Simple proton spectroscopic imaging. Radiology 153: 189-194, 1984

18. Bol GH, Kotte AN, van der Heide UA and Lagendijk JJ: Simultaneous multi-modality ROI delineation in clinical practice. Comput Methods Programs Biomed 96: 133-140, 2009.

19. Smitt MC, Birdwell RL and Goffinet DR: Breast electron boost planning: Comparison of CT and US. Radiology 219: 203-206, 2001.

20. Kirby AM, Yarnold JR, Evans PM, Morgan VA, Schmidt MA, Scurr ED and desouza NM: Tumor bed delineation for partial breast and breast boost radiotherapy planned in the prone position: What does MRI add to X-ray CT localization of titanium clips placed in the excision cavity wall? Int J Radiat Oncol Biol Phys 74: 1276-1282, 2009

21. den Hartogh MD, van den Bongard HJ, Davidson MT, Kotte AN, Verkooijen HM, Philippens ME, van Vulpen M, van Asselen B and Pignol JP: Full-thickness closure in breast-conserving surgery: The impact on radiotherapy target definition for boost and partial breast irradiation. A multimodality image evaluation. Ann Surg Oncol 21: 3774-3779, 2014.
22. Giezen M, Kouwenhoven E, Scholten AN, Coerkamp EG, Heijenbrok M, Jansen WP, Mast ME, Petoukhova AL and Struikmans H: MRI- versus CT-based volume delineation of lumpectomy cavity in supine position in breast-conserving therapy: An exploratory study. Int J Radiat Oncol Biol Phys 82: 1332-1340, 2012

23. Jolicoeur M, Racine ML, Trop I, Hathout L, Nguyen D, Derashodian T and David S: Localization of the surgical bed using supine magnetic resonance and computed tomography scan fusion for planification of breast interstitial brachytherapy. Radiother Oncol 100: 480-484, 2011.

24. Kirby AN, Jena R, Harris EJ, Evans PM, Crowley C, Gregory DL and Coles CE: Tumour bed delineation for partial breast/breast boost radiotherapy: What is the optimal number of implanted markers? Radiother Oncol 106: 231-235, 2013.

25. Palta M, Yoo S, Adamson JD, Prosnitz LR and Horton JK: Preoperative single fraction partial breast radiotherapy for early-stage breast cancer. Int J Radiat Oncol Biol Phys 82: 37-42, 2012.

26. Nichols EM, Feigenberg SJ, Marter K, Cheston SB, Lasio G, Tkaczuk K, Kesmodel S, Buras R and Regine WF: Preoperative radiation therapy significantly increases patient eligibility for accelerated partial breast irradiation using 3D-conformal radiotherapy. Am J Clin Oncol 36: 232-238, 2013.

27. van der Leij F, Elkhuizen PH, Janssen TM, Poortmans P, van der Sangen M, Scholten AN, van Vliet-Vroegindeweij C and Boersma LJ: Target volume delineation in external beam partial breast irradiation: Less inter-observer variation with preoperative- compared to postoperative delineation. Radiother Oncol 110: 467-470, 2014.

28. Schmitz AC, van den Bosch MA, Loo CE, Mali WP, Bartelink H, Gertenbach M, Holland R, Peterse JL, Rutgers EJ and Gilhuijs KG: Precise correlation between MRI and histopathology - exploring treatment margins for MRI-guided localized breast cancer therapy. Radiother Oncol 97: 225-232, 2010.

29. den HartoghMD: TowardsMRI-guided radiotherapyinearly-stage breast cancer patients. https://www.umcutrecht.nl/getmedia/ b6fd022c-ffc9-49d0-a26f-602916d5d225/Proefschrift MdenHartogh.pdf.aspx. PhD dissertation, University Medical Center Utrecht. Utrecht, The Netherlands, 2014. 